\title{
Regional North American Annual Meeting of the World Federation of Neurology - Research Group on Neuroepidemiology
}

Northwestern University, Chicago, III., April 18, 2008

\section{Program and Abstracts}

Guest Editors

Teepu Siddique, Chicago, III.

Amethyst Leimpeter, Oakland, Calif.

Stephen K. Van Den Eeden, Oakland, Calif. 


\section{Program}

Abstract No.

\begin{tabular}{|c|c|c|}
\hline $\begin{array}{l}\text { 8:00 AM } \\
8: 45\end{array}$ & $\begin{array}{l}\text { Registration opens } \\
\text { Poster Set-up and Viewing }\end{array}$ & P1-P9 \\
\hline $9: 15$ & Introduction & \\
\hline 9:35 & $\begin{array}{l}\text { Prognostic Predictors of Late Remission in Epilepsy } \\
\text { E. Beghi }\end{array}$ & 1 \\
\hline $9: 55$ & $\begin{array}{l}\text { Active and Passive Smoking and Risk of Narcolepsy in People with HLA DQB1*0602 } \\
\text { T. Ton }\end{array}$ & 2 \\
\hline $10: 15$ & $\begin{array}{l}\text { Evidence for Association between Calcium-Related Genes and Successful Cognitive Aging } \\
\text { S. Edland }\end{array}$ & 3 \\
\hline 10:35 & Break & \\
\hline 11:05 & $\begin{array}{l}\text { A Case-Control Study on Coffee Drinking, Caffeine-Related Genes, and Parkinson's Disease } \\
\text { M. Facheris }\end{array}$ & 4 \\
\hline $11: 25$ & $\begin{array}{l}\text { Polymorphisms in Vitamin D Metabolism Genes and Risk of Multiple Sclerosis } \\
\text { K. Simon }\end{array}$ & 5 \\
\hline $11: 45$ & Business Meeting & \\
\hline 12:15 PM & Lunch & \\
\hline $12: 45$ & Poster Viewing & P1-P9 \\
\hline $12: 55$ & $\begin{array}{l}\text { Keynote Address - Genetic Etiologies Implicate Environmental Factors in Motoneuron } \\
\text { Degeneration } \\
\text { T. Siddique }\end{array}$ & \\
\hline $1: 55$ & $\begin{array}{l}\text { Physical Activity, Nutrition and Alzheimer's Disease } \\
\text { N. Scarmeas }\end{array}$ & 6 \\
\hline $2: 15$ & $\begin{array}{l}\text { Cancer and Blood Concentrations of the Co-Mutagen Harmane in Essential Tremor } \\
\text { E. Louis }\end{array}$ & 7 \\
\hline $2: 35$ & Final Comments and Adjournment & \\
\hline
\end{tabular}




\section{Oral Presentations}

\section{1}

\section{Prognostic Predictors of Late Remission in Epilepsy}

E. Beghi, L. Specchio, A. Del Felice, G. Boero, G. Bogliun, A. La Neve, A. De Palo

Milano, Foggia, Verona, Taranto, Monza, Bari, Italy

Objective: To define the proportion of patients with newly diagnosed epilepsy entering early and late remission and to identify prognostic predictors. Background: Patients with newly diagnosed epilepsy entering seizure remission are $55-69 \%$ in population-based studies. Although most remissions occur early in the course of the disease, late remissions may also occur after prolonged periods of follow-up. Data on late remissions are scarce and prognostic predictors are virtually unknown. Methods: 174 women and 178 men (mean age 31.5 years) with newly diagnosed epilepsy were recruited in two epilepsy centers (Monza, Bari) and followed for 2399.6 person-years (mean 82.7 months; median 75.0 months). For each eligible case, the following prognostic predictors were identified: age, gender, putative etiology, first EEG record, neurological and psychiatric examination, disease duration at diagnosis, seizure type(s) and number prior to starting treatment, epilepsy syndrome, and first antiepileptic drug. A period of remission was defined by achieving 2 or more years of complete seizure control after treatment start. The 2 -year cumulative timedependent probability of remission was calculated with the Kaplan-Meier method. Early remission was defined by a 2 -year seizure control achieved immediately after treatment start. Late remission was defined by a 2-year seizure control achieved at least 24 months after treatment start. Prognostic predictors were assessed by binary logistic regression analysis with forward stepwise likelihood ratio, adjusting for center. Results: 85 patients (23.0\%) achieved early remission and 40 patients (10.8\%) achieved late remission. The cumulative time-dependent probability of 2 year remission was $26.1 \%$ at 2 years after treatment start, and 53.9 , 75.0 and $77.5 \%$ at 4,7 and 10 years. Younger age was the only independent predictor of late remission. For each increasing year of age there was a 0.97 decrease in the risk of late remission $(95 \% \mathrm{CI}$ 0.94-0.99). Conclusion: Late remission tends to occur in more than $10 \%$ of patients with newly diagnosed epilepsy followed for a prolonged period of time. Younger age is the only predictor of late remission.

\section{2 \\ Active and Passive Smoking and Risk of Narcolepsy in People with HLA DQB1*0602}

T. Ton, W. Longstreth, Jr., T. Koepsell

Seattle, Wash., USA

Objective: To examine the relation between active and passive smoking and risk of narcolepsy among genetically susceptible individuals. Background: Currently lacking are reports examining the association between smoking and risk of narcolepsy, a sleep disorder characterized by excessive daytime sleepiness and cataplexy, episodic weakness triggered by strong emotions. One etiologic model proposes that environmental factors selectively and irreversibly destroy hypocretin-producing cells in the lateral hypothalamus in genetically susceptible individuals with HLA $\mathrm{DQB}^{*} 0602$. The association between exposure to active and passive smoking and narcolepsy risk in susceptible individuals is unknown. Methods: We conducted a population-based case-control study in King County, Washington. Between 2001-2005, 67 prevalent cases were enrolled in the study, and 95 controls were recruited through random-digit dialing. Subjects were between ages $18-50$ and positive for HLA DQB1 ${ }^{*} 0602$. All were administered in-person interviews about their history of active and passive smoking. Results: An increased risk of narcolepsy was associated with having lived with two or more household smokers (odds ratio $[\mathrm{OR}]=5.1 ; 95 \%$ confidence interval $[\mathrm{CI}]: 1.6,12.1)$; having lived with a grandparent or a sibling who smoked $(\mathrm{OR}=3.0 ; 95 \%$ CI: 1.1, 8.3); having lived with a non-family household member who smoked $(\mathrm{OR}=3.7 ; 95 \% \mathrm{CI}$ : 1.6, 8.6); and having lived with an unrelated smoker for two or more years $(\mathrm{OR}=2.9$; $95 \% \mathrm{CI}: 1.1$, 7.8). The risk of narcolepsy was not associated with exposure to smoke at work or with active smoking before age 21 or before age of narcolepsy onset. Conclusions: Passive smoking may be more influential than active smoking given that symptoms of narcolepsy usually manifest themselves in adolescence and that exposure in childhood to passive smoke occurs more commonly than to active smoke. Future studies could help verify whether passive smoking is an important etiologic component of narcolepsy among genetically susceptible individuals.

03

Evidence for Association between Calcium-Related Genes and Successful Cognitive Aging

S. Edland, D. Von Muhlen, G. Laughlin, D. Kritz-Silverstein, E. Barrett-Connor

San Diego, Calif., USA

Objective: To test the association between a panel of calciumrelated single nucleotide polymorphisms (SNPs) and cognitive decline while maintaining an experiment-wide alpha error rate of five percent. Background: Calcium-related genes may be implicated in age-associated cognitive decline. Testing multiple genetic associations with Bonferroni correction is statistically inefficient and unlikely to detect associations with polygenetic traits determined by multiple genes with small contributions from each gene. Methods: Nine hundred and twenty-four persons in the Rancho Bernardo Study received the Mini-Mental State exam (MMSE), a brief global cognitive function scale, in 1988 and again approximately four years later (60\% female; age 50 to 92 years, MMSE 17 to 30 at first cognitive exam). The MMSE change outcome variable was dichotomized at the median into improvers (47\%) and nonimprovers (53\%). Thirty-eight potentially functional SNPs on 23 genes were chosen based on gene function or previously reported association with osteoporosis. $\mathrm{p}$ values for 
each SNP were calculated separately for men and women by a logistic regression allelic score test after controlling for age. Under the null hypothesis of no association the distribution of $p$ values would be uniform with an expected mean $p$ value of 0.5 . We tested this null against the alternative hypothesis of a mean $p$ value less than 0.5 using Wilcoxon's signed rank test. Results: The mean $\mathrm{p}$ value of 0.43 was statistically significantly less than expected mean under the null (Wilcoxon's $\mathrm{V}=1,038$, experiment-wise $\mathrm{p}=$ 0.014). A post-hoc analysis found that nine percent of the SNP associations tested were significant at the 0.05 level, about twice what would be expected due to random chance. Conclusions: These data are consistent with the involvement of calcium-related processes in age-associated cognitive decline. Experiment-wise hypothesis testing may provide a statistically efficient way of testing mechanistically related genes for association with phenotype or disease.

\section{4 \\ A Case-Control Study on Coffee Drinking, Caffeine-Related Genes, and Parkinson's Disease}

M. Facheris, N. Schneider, T. Lesnick, M. Andrade, J. Cunningham, W. Rocca, D. Maraganore

Rochester, Minn., USA

Objective: To study the association of coffee and caffeine-related genes with Parkinson's disease (PD). Background: Coffee drinking may be inversely associated with $\mathrm{PD}$, possibly due to a neuroprotective effect of caffeine. The individual variability in the effects of coffee raises the possibility of gene-environment interactions. Methods: We included 1,208 subjects (446 case-unaffected sibling pairs and 158 case-unrelated control pairs), primarily Caucasian and of European ancestry, residing in the Upper Midwest (USA), and with available coffee and genotype data. We collected information on coffee drinking (ever/never and cups per day) via structured telephone interview. We studied two genes: $C Y P 1 A 2$, which encodes the major rate-limiting step of caffeine metabolism (variants rs35694136 and rs762551); and ADORA2A, which encodes the major receptor activity of caffeine in the brain (variants rs5751876 and rs3032740). We confirmed Hardy-Weinberg equilibrium for the four variants in controls, and employed data from a genome-wide association study of PD and from this study to construct linkage disequilibrium maps for the two genes. Conditional logistic regression models were used to study the association of coffee and the four gene variants with PD susceptibility, and Cox proportional hazard models to study age at onset. Analyses were stratified by type of case-control pair, age at study, sex, and family history of PD. Results: We did not observe significant main or joint associations of coffee drinking or of the genetic variants with PD susceptibility, overall and in most strata. While coffee drinking was associated with later age at onset of PD (cases only analyses), this finding could be explained by a secular trend of reduced coffee drinking (as observed in controls). Conclusions: Our study does not support the hypothesis that coffee protects against PD, nor does it provide evidence for a gene-environment interaction.

\section{5 \\ Polymorphisms in Vitamin D Metabolism Genes and Risk of Multiple Sclerosis}

\author{
K. Simon, X. Yang, K. Munger, A. Ascherio
}

Boston, Mass., USA

Objective: To determine whether polymorphisms in genes associated with vitamin D metabolism are associated with risk of multiple sclerosis (MS). Background: Several lines of evidence support a role for vitamin D in multiple sclerosis (MS). Therefore, polymorphisms in genes that affect vitamin $\mathrm{D}$ metabolites are good candidates for association with MS. Methods: We conducted a nested case-control study among participants in the Nurses' Health Study and Nurses' Health Study II. A total of 214 MS cases with biological samples were included in the main analyses. For each case, two controls matched by year of birth were randomly selected from cohort participants. Previously reported SNPs in VDR, CYP27B1, CYP24A1, CYP2R1 and DBP were chosen based on potential functional impact and minor allele frequency $(>10 \%)$. Conditional logistic regression models were used to calculate relative risks of MS according to genotype. Further, we assessed whether or not these SNPs modified previously observed associations with latitude of residence and vitamin D intake. Results: None of the SNPs genotyped were associated with risk of MS. However, a significant interaction was observed between vitamin D intake and the VDR_FokI polymorphism $(\mathrm{p}=0.02)$. Amongst individuals with the 'FF' genotype, no association between vitamin $\mathrm{D}$ and risk of $\mathrm{MS}$ was observed ( $\mathrm{RR}=1.08,95 \%$ $\mathrm{CI}=0.51,2.31$; for $400 \mathrm{IU} /$ day increase), whereas, amongst those with the 'ff' genotype, an $80 \%$ decreased risk of MS was observed $(\mathrm{RR}=0.21,95 \% \mathrm{CI}=0.04,1.22$; for $400 \mathrm{IU} /$ day increase). Similarly, although not significant, the effect of increased MS risk associated with increasing latitude was stronger amongst women with the 'ff' genotype. Conclusions: These results do not support a strong association between the genotyped vitamin D related SNPs and risk of MS. However, in our small sample, there was a suggestion of a potential interaction between environmental exposure to vitamin D and the VDR_FokI genotype.

\section{6}

\section{Physical Activity, Nutrition and Alzheimer's Disease}

N. Scarmeas, Y. Stern, J. Luchsinger

New York, N.Y., USA,

Objective: To investigate the combined effect of nutrition and physical exercise in Alzheimer's disease (AD). Background: Both higher Mediterranean Diet (MeDi) adherence and physical activity (PA) have been associated with decreased AD risk. Methods: A subset of a community cohort of elderly in New York had evaluations of both diet (adherence to the MeDi; categorized as lowmedium-high) and PA (number of minutes of weekly participation in [light, moderate or severe] physical activities; categorized as low-medium-high). MeDi, $\mathrm{PH}$ and a 3 level variable combining MeDi and PA information (MeDi-PA: low-low vs. low-high/high- 
low vs. high-high) were used as predictors in logistic regression models. After evaluation with standardized neurological and neuropsychological measures 104 subjects were diagnosed with $\mathrm{AD}$ at the baseline evaluation, while 1,041 subjects received a nondementia diagnosis. AD vs. non-dementia status served as the outcome. Models were adjusted for cohort (years 1992 vs. 1999), age, gender, ethnicity, education, APOE genotype, a comorbidity index, caloric intake and body mass index. Results: Both MeDi adherence and $\mathrm{PH}$ were associated with lower $\mathrm{AD}$ odds when considered simultaneously in a single model: MeDi middle adherence OR 0.47 [0.23-0.94], high adherence OR 0.25 [0.11-0.57] $\mathrm{p}$ for trend $=0.001$; PA middle OR $0.68[0.33-1.40]$; high OR $0.46[0.21-$ $1.02] ; \mathrm{p}=0.05)$. When the variable combining MeDi and PA was considered, as compared to subjects in the low-low MeDi-PA category, ORs for AD were as follows: low-high/high-low MeDi-PA 0.48 (0.23 - 0.95), high-high MeDi-PA 0.28 (0.12 - 0.68); p for trend $=0.007$. Conclusions: Adoption of both increased physical activity and healthy nutrition behaviors seem to be associated with lower odds for $\mathrm{AD}$, in an independent manner.

\section{7}

\section{Cancer and Blood Concentrations of the Co-Mutagen Harmane in Essential Tremor}

E. Louis, K. Pellegrino, P. Factor-Litvak, E. Rios, W. Jiang, C. Henchcliffe, W. Zheng

New York, N.Y., West Lafayette, Ind., USA

Objective: To (1) compare the prevalence of cancer in essential tremor (ET) cases vs. controls, and (2) determine whether blood harmane concentrations are elevated among ET cases with cancer. Background: Harmane (1-methyl-9H-pyrido[3,4- $b$ ]indole) is a potent tremor-producing dietary neurotoxin. Blood concentrations are elevated in ET. Harmane is also a co-mutagen. Medical co-morbidity has not been studied extensively in ET; there are no data on the prevalence of cancer in ET. Methods: Participants were enrolled in a cross-sectional study of the environmental epidemiology of ET. Cancer, reported by participants, was confirmed by a review of medical records and pathology reports. Blood harmane concentration was quantified using high performance liquid chromatography. Results: The 267 ET cases and 331 controls were similar in terms of demographic characteristics. Sixtysix (24.7\%) ET cases vs. 55 (16.6\%) controls had cancer (adjusted $\mathrm{OR}=1.52,95 \% \mathrm{CI}=1.01-2.30, \mathrm{p}=0.04)$. Among specific cancer types, colon cancer was more prevalent in ET cases than controls ( $2.6 \%$ vs. $0.6 \%, p=0.04)$. Log blood harmane concentration was higher in ET cases vs. controls $\left(0.61 \pm 0.63 \mathrm{~g}^{-10} / \mathrm{ml}\right.$ vs. $0.44 \pm$ $\left.0.68 \mathrm{~g}^{-10} / \mathrm{ml}, \mathrm{p}=0.02\right)$ and in participants with vs. without cancer $\left(0.70 \pm 0.68 \mathrm{~g}^{-10} / \mathrm{ml}\right.$ vs. $\left.0.48 \pm 0.65 \mathrm{~g}^{-10} / \mathrm{ml}, \mathrm{p}=0.02\right)$. Log blood harmane concentration was highest in ET cases with cancer $(0.87$ $\left.\pm 0.68 \mathrm{~g}^{-10} / \mathrm{ml}\right)$ when compared with other groups $(\mathrm{p}=0.009)$. Conclusions: Harmane is both tremorogenic and co-mutagenic, so that it is possible that elevated blood harmane concentration is a common determinant for both diseases (i.e., ET and cancer). One possibility is that differences in dietary harmane intake predispose to these diseases. Another possibility is that genetic differences lead to differences in the ability to metabolize dietary harmane. Each of these models requires further exploration.

\section{Poster Presentations}

\section{P 1 \\ Disability and Survival of Multiple Sclerosis in Saskatoon Saskatchewan - A Thirty Year Follow-Up \\ W. Hader}

Saskatoon, Sask., Canada

Background: A population-based cohort prevalent group of 150 clinical definite patients ascertained on 1 January 1977, in the city of Saskatoon Saskatchewan, was followed for thirty years. Objective: To outline the clinical characteristics, compare the levels of disability at $15,25,35,45$ years after onset, and to estimate the duration of disease and survival. Methods: Clinical records were maintained, with the cohort review each decade for thirty years. The levels of disability according to the Kurtzke Disability Status Scale and survival times were recorded. SPSS and Kaplan-Meier methods were used for analysis. Results: On prevalence day there were 48 men and 102 women, average age of onset $32.1 \pm 10$ years and $28.5 \pm 8.6$ years. The average duration of disease was 15.7 years. On 1 January 2007, 39 (26\%) patients were living, 105 (70\%) deceased, and 6 (4\%) unable to locate. The mean age of the 29 living women was $65.9 \pm 9.7$ years, and the 10 men $68.2 \pm 8.2$ years. The mean duration of disease is $40.4 \pm 7.2$ years for women, and $41 \pm 4.0$ years for men. The disabilities compared between 1977 and 2007, indicates at 15 and 45 years after onset, the EDSS $\leq 2.5$ was 33.3 and $8 \%$, mild $3-5.5$ was 17.3 and $2.7 \%$, moderate $6-7.5$ was 26.6 and $4.7 \%$, severe, 22.7 and $10.7 \%$. The median survival time after onset was 33 years for men and 38 years for women. The mean age of death for the 67 women was $63.3 \pm 13.6$ years, and $64.2 \pm 14$ years for the men. The median duration of life was 69.1 years for men and 68.5 years for women. Conclusions: Multiple Sclerosis is a progressive neurological disorder and long-term survival is associated with moderate to severe disability and decreased life expectancy in this natural history study.

\section{P 2 \\ Population-Based Prospective Study of Cigarette Smoking and Risk of Incident Essential Tremor}

E. Louis, J. Benito-León, F. Bermejo-Pareja

New York, N.Y., USA, Madrid, Spain

Objective: Utilizing prospective, longitudinal data, we tested the hypothesis that cigarette smoking at baseline was associated with reduced risk of incident essential tremor (ET) at follow-up. Background: Smoking cigarettes is associated with lower risk of Parkinson's disease (PD). Despite the established clinical links between PD and ET, there are virtually no data on smoking in ET. One study showed an association between smoking and lower prevalence of ET. However, given its cross-sectional design, it was unclear whether a reduction in cigarette smoking preceded or merely followed the onset of tremor (e.g., perhaps in an attempt 
to reduce nicotine-associated tremor). Methods: Using a population-based, cohort design, baseline cigarette smoking habits were assessed in 3,348 participants in an epidemiological study in central Spain, among whom 77 developed incident ET. Results: There were 3,348 participants, among whom 397 (11.9\%) were current smokers at baseline. Five (6.5\%) of 77 incident ET cases had been smokers at baseline compared with 392 (12.0\%) of 3,271 controls $(\mathrm{p}=0.14)$. Baseline pack-years were lower in incident ET cases than controls $(9.2 \pm 17.7$ vs. $15.7 \pm 28.4, \mathrm{p}=0.002)$. Participants were stratified into baseline pack-year tertiles and few incident ET cases were in the highest tertile (4 [5.2\%] cases vs. 431 [13.2\%] controls, $\mathrm{p}=0.039$ ). In Cox Proportional Hazards Models, highest baseline pack-year tertile was associated with lower risk of incident ET; those in the highest pack-year tertile were one-third as likely to develop ET when compared to non-smokers $(\mathrm{RR}=0.29$, 95\% CI $=0.09-0.90, \mathrm{p}=0.03$ [adjusted model]). Conclusions: Smoking has been reported to play a role in several neurodegenerative diseases, including PD and multiple system atrophy. We demonstrated an association between baseline heavy cigarette smoking and lower risk of incident ET. The biological/toxicological basis for this association merits additional investigation.

\section{P 3}

Care of Stroke Patients in Danang Province, Vietnam

\section{A. Fitzpatrick, Q. Ngo, T. Lam, D. Tirschwell, T. Ton}

Seattle, Wash., USA, Danang, Vietnam

Objective: To identify points of initial contact between stroke patients and physicians in Danang, Vietnam. Background: Worldwide, a shift in health care manpower needs is occurring in developing countries as they experience a health transition from infectious to chronic diseases Chronic diseases increase the burden on health care systems in these countries. The flow of care from stroke onset to treatment has not been previously described in Danang, Vietnam. Methods: Interviews with heads of departments at Danang Hospital provided information on patterns of care for incoming stroke patients. Results: Danang has a population of 803,853 . In 2002, over 1,200 hospitalizations for cerebrovascular disease were reported to the Danang Department of Health. Of these, $18 \%$ were classified as 'cerebral hemorrhage', $18 \%$ as 'cerebral vascular embolism', $23 \%$ as stroke of an 'undefined cause', and $41 \%$ as 'other cerebral vascular diseases'. Almost all cases are eventually seen at Danang Hospital either as self-referrals or referred from one of the nine outlying hospitals. Usually admitted through the Emergency Department, critical cases with coma, respiratory failure, or cardiac failure, are treated in the ICU. Majority of cases are triaged to care in the Cardiovascular Internal Medicine Department. Patients with indications for surgery are sent to the Neurologic Internal Medicine or Surgical Departments. Following acute treatment, patients are referred to the Department of Rehabilitation within the Hospital or to the outpatient Rehabilitation Center. Those with cognitive problems are referred to the Psychiatric Hospital. Compliance for follow-up rehabilitation is low. Currently, Danang has a total of four neurologists and 26 psychiatrists. Conclusions: Identification and treatment of stroke patients in Danang occurs within a general medi- cal or cardiology setting rather than in specialty stroke units. Recommendations for rehabilitation are infrequently followed. The need to develop capabilities and resources here in stroke care is great.

\section{P 4 \\ Do Head Injury or Cigarette Smoking Contribute to the Increased Risk of Amyotrophic Lateral Sclerosis in US Veterans?}

\author{
S. Schmidt, K. Allen, J. Rimmler, V. Loiacono, C. Stanwyck, L. Nelson, \\ E. Oddone
}

Durham, N.C., Palo Alto, Calif., USA

Objective: An increased risk of amyotrophic lateral sclerosis (ALS) has been reported among US military veterans. A range of environmental factors with higher exposure probabilities for military personnel, compared to civilians, may contribute to this observation. These factors include head injury and cigarette smoking, which have previously been associated with ALS risk in some, but not all epidemiologic studies. Methods: In conjunction with the National Registry of Veterans with ALS, we have enrolled 578 cases and 366 controls into the GENEVA case-control study. We have analyzed the relationship between head injury, cigarette smoking and ALS risk in the entire study sample and a subset of incident cases ( $\mathrm{n}=194,34 \%)$. Results: The number of head injuries that required medical attention was not significantly associated with ALS risk. However, an older age at the time of the last head injury was associated with an elevated risk, relative to those who never suffered a head injury, with an odds ratio (OR) of 1.8 $(95 \%$ CI $0.8,3.9)$ for an age at last injury of $30-40$ years, and an OR of $2.1(95 \%$ CI 1.1, 4.2) for an age at last injury of $>40$ years ( $\mathrm{p}=0.03$ for the subgroup of incident cases). There was no significant association between different measures of lifetime cigarette smoking history and ALS risk. The majority of the reported head injuries occurred outside the veterans' service periods. However, more veterans with an older age at the most recent head injury had been deployed to a major conflict and/or had received imminent danger pay, compared to those who never suffered a head injury. Conclusions: We conclude that a higher frequency of head injury may make a moderate contribution to the increased risk of ALS in US veterans.

\section{P 5 \\ Reliability of Caregiver Assisted Telephone Interviews of Dysarthric ALS Patients}

M. Kilty, X. Liu, D. Bell, J. Sandner, P. Factor-Litvak, H. Mitsumoto

New York, N.Y., USA

Objective: To determine the reliability of telephone interviews of dysarthric patients, using caregivers to facilitate communication with the interviewer. Background: Approximately $25 \%$ of 
ALS patients first present with bulbar symptoms resulting in dysarthria. Other patients develop dysarthria during the disease course. Significant dysarthria makes telephone interviews of patients impossible. This problem exists in any study utilizing telephone interviews, especially prospective studies. Using a caregiver to facilitate responses from a patient may help resolve this situation. Methods: A short telephone interview was developed with items on recent exposures. These included exercise, diet, occupation, hobbies, and PHQ-9, a depression scale. A convenience sample of patients without dysarthria and their caregivers was selected for interview. ALS patients were interviewed first without the caregiver present. Within one week, caregivers were interviewed in the presence of the patient. Caregivers were asked to consult with patients on responses. To simulate the situation of a dysarthric patient with advanced disease, patients were instructed to use non-verbal gestures (e.g. nodding, hand gestures) in communicating with their caregiver. Reliability of items between the patient and caregiver pairs was assessed using kappa and ICC, as appropriate. Results: Most items had good levels of reliability. For hobbies and occupation, most kappas were over 0.80 . On the PHQ-9, 4 items had ICCs over $0.75,4$ ranged from 0.48 to 0.60 and one had poor agreement. Results for diet and physical activity were mixed. The study process also suggested some changes to improve reliability in future studies, including revision of certain questions and of caregiver instructions. Conclusions: These results indicate good reliability between the caregiver-assisted interview and the patient's responses suggesting that caregiver-assisted interviews can be useful in ALS studies.

\section{P 6}

\section{Statin Use Prior to Amyotrophic Lateral Sclerosis (ALS) and Primary Lateral Sclerosis (PLS)}

B. Brooks

Charlotte, N.C., USA

Objective: Define antecedent statin use in patient populations attending an ALS Clinic. Background: Statin use, including red yeast rice, has been rarely associated with myopathy and other neuromuscular symptoms. Some patients referred to an ALS Clinic have described muscle pain and weakness following initiation of statins leading to the diagnosis of ALS. Methods: Review of prevalence of statin use in 240 [M-153; F-87] sporadic [s] or familial [f] patients with ALS or other motor neuron disease syndromes [MNDs] seen in a Midwest university-associated ALS Clinic. Results: Among 31/164 statin-exposed sALS patients, 11 single statin treated [SST] and 3 multiple statin treated [MST] patients described treatment-associated pain and increased weakness before diagnosis. Only those 3 MST patients have been reported to MedWatch. In 13 sALS patients requiring thyroid [T] replacement, 1/3 SST and 2/2 MST patients had symptoms prior to ALS diagnosis. In $7 \mathrm{sALS}$ patients with diabetes mellitus [DM] treated with oral agents, none required T but 1/3 SST and 1/1 MST patients had symptoms. In 4 sALS patients with DM requiring insulin, $2 / 4$ required $\mathrm{T}$ but $0 / 2$ SST and $1 / 2$ MST patients had symptoms prior to diagnosis. Among $18 \mathrm{fALS}$ patients none had
DM or T and 0/2 SST patients had symptoms. Among 20 PLS patients none had DM or T and 1/2 SST and 1/1 MST patients had symptoms. There was no statin or T use, or DM among $14 \mathrm{dysim-}$ mune motor neuropathy/neuron disease or atypical MNDs prior to diagnosis. Conclusions: Statin-associated pain and weakness is more common in MST sALS, hypothyroid sALS and DM sALS patients than fALS or other MNDs patients. Further studies are required to determine if statin use, particulary MST in the context of changing statins in response to statin-related symptoms is a trigger for sALS in some susceptible patients.

\section{P 7 \\ Prevalence of Narcolepsy in King County, Washington}

W. Longstreth, Jr., T. Ton, T. Koepsell, V. Gersuk, A. Hendrickson, S. Velde

Seattle, Wash., USA

Objective: To estimate the prevalence of physician-diagnosed narcolepsy and to identify patients for future epidemiology studies. Background: Population-based studies pose many challenges for diseases that rarely result in hospitalization or death and for which few if any pre-existing registries exist. Population-based investigations of uncommon diseases, such as narcolepsy, are essential if we are to learn more about its etiology and prevention. Few epidemiologic studies have focused on narcolepsy, a disabling sleep disorder characterized by excessive daytime sleepiness, episodic loss of muscle tone with strong emotions (cataplexy) and association with human leukocyte antigen (HLA-) DQB1*0602. Methods: We used multiple overlapping methods to identify residents of a specific population who were 18 years or older with physician-diagnosed narcolepsy in King County, Washington. These patients were entered into a registry and recruited into an epidemiologic study that entailed an interview and buccal scrapings to determine HLA-DQB1*0602 status. Results: Missing values were imputed to allow prevalence to be estimated based on all 425 patients entered into the registry between 2001 and 2005, whether they were recruited into the epidemiologic study $(n=279)$ or not $(n=146)$. As of 2001 July 01 , the estimated prevalence per 100,000 of physician-diagnosed narcolepsy with cataplexy was 21.8 (95\% confidence interval (CI): 18.8, 24.8), in the range of what has been reported in prior studies. The median age of onset was 14 (interquartile range: 10, 18). For narcolepsy with or without cataplexy, prevalence was 30.6 (95\% CI: 27.6, $33.5)$, and for narcolepsy with HLA-DQB1 ${ }^{*} 0602$, prevalence was 15.3 (95\% CI: 12.8, 17.9). Estimated prevalence was higher in women than men and in African-Americans than other racial groups. Conclusions: These differences could reflect problems in identification and recruitment or may provide etiologic clues about narcolepsy. This study illustrates the challenges in performing population-based studies of narcolepsy. 


\section{P 8}

\section{Prevalence of Epilepsy in Danang, Vietnam}

T. Ton, T. Lam, Q. Ngo, A. Fitzpatrick, W. Longstreth, Jr., J. Zunt

Seattle, Wash., USA, Danang, Vietnam

Objective: To describe the epilepsy registry and to estimate the prevalence of epilepsy in Danang, Vietnam. Background: In China and Laos, the prevalence of epilepsy ranges from 4 to 8 per 1,000 . Awareness of epilepsy is limited, and published prevalence estimates are unavailable for Vietnam. Danang is a central province in Vietnam with one Psychiatric Hospital serving 803,853 people. Unlike in the US, all patients in Vietnam with seizures and epilepsy are referred to and treated by psychiatrists. The Psychiatric Hospital maintains a registry of patients with epilepsy in Danang. Methods: We used descriptive statistics to explore characteristics of patients in the registry. The prevalence of epilepsy in each of the seven districts was obtained by dividing the number of cases within each district by the district population in 2007. Confidence intervals were obtained assuming a Poisson distribution. Results: The registry contains 1,585 patients. The average age of patients in the registry is 29.4 years. The prevalence in the seven districts ranged from 0.6 per 1,000 (95\% CI: $0.5,0.8)$ in Hoa Vang district to 2.1 per 1,000 (95\% CI: $1.8,2.5)$ in Lien Chieu district. The overall prevalence of epilepsy in Danang is 2.0 per 1,000 (95\% CI: 1.4 1.6). Conclusions: The prevalence of epilepsy in Danang was at least half that reported in China and Laos. We suspect underestimation may be due to the registry including only the most severe cases that were more likely to come to medical attention or perhaps lack of recognition of epilepsy in communities or health posts. Financial and other barriers to seeking medical care may also contribute to the underestimation. A door-to-door survey is planned to obtain a valid prevalence of epilepsy in Danang and to better understand how medical services are accessed by people with epilepsy.

\section{P 9 \\ Blood Metal Levels in Parkinson's Disease (PD) \\ J. Hoppin, F. Kamel, M. Korell, D. Umbach, G. Ross, S. Goldman, G. Bhudhikanok, A. Blair, D. Sandler, C. Tanner \\ Research Triangle Park, N.C., Sunnyvale, Calif., USA}

Objective: To compare blood metal levels in PD cases and controls. Background: Metals have been implicated as risk factors for $\mathrm{PD}$, and one study reported higher whole blood levels of copper, iron, magnesium, and zinc in PD cases compared to controls. Methods: In the FAME study, a case-control study of PD nested in the Agricultural Health Study, we measured whole blood metal levels using inductively coupled plasma-mass spectrometry in 99 confirmed PD cases and 116 matched controls. Samples were collected in trace metal free vacutainers during home visits to farms in Iowa and North Carolina between 2002-2006. The mean time between diagnosis and home visit was 7.4 years. Results: Seven of ten elements analyzed were detected in almost all subjects (copper, iron, lead, magnesium, manganese, selenium, and zinc). Although we found expected associations of metals with known predictors (e.g., lead and smoking, lead and age), we observed no differences in blood metal levels between PD cases and controls. Further, the values that we found for both cases and controls were similar to those reported for cases in the previous study. Among cases, we saw no differences related to time since diagnosis. The half-life of metals in blood is relatively short, and the latency between metal exposure and disease incidence may be long. Thus current blood levels may not reflect the etiologically relevant time-window. Conclusions: Metal levels in samples collected from cases after PD diagnosis do not differ from levels in controls. 